\title{
Modelling ad-hoc DRT over many days: a preliminary study
}

\author{
$\underline{\text { N. Ronald }}^{\mathrm{ab}}$, R. Thompson ${ }^{\mathrm{a}}$ and S. Winter ${ }^{\mathrm{a}}$ \\ ${ }^{a}$ Department of Infrastructure Engineering, The University of Melbourne, 3010, Australia \\ ${ }^{\mathrm{b}}$ Faculty of Science, Engineering and Technology, Swinburne University of Technology, Hawthorn, 3122, \\ Australia \\ Email: $\underline{\text { ronald@swin.edu.au }}$
}

\begin{abstract}
Traditional transport models focus on commuter traffic in private vehicles or public transport. However, new methods of travel are gaining traction, both in discussions and implementation: carsharing, shared taxis/buses, autonomous vehicles. Modelling these requires more attention on the interaction between people and place than traditional modes.
\end{abstract}

As part of a larger project on mobility on demand, shared flexibly-scheduled vehicles (also known as demandresponsive transportation, DRT) have been explored using simulations. These schemes can be designed in different ways with respect to the resolution of stops and timings, and the potential patronage. In a pure adhoc scheme, there will be no timetable or route. These schemes are attractive in areas where mass transit cannot provide a reasonable level-of-service in a financially and environmentally sustainable manner, such as fringe areas and small towns, however could also find a niche providing access to mass transit in larger cities.

One still-outstanding problem is predicting demand for DRT. Previous experiments have focused on trips, but have rarely looked into the choice to (continue to) use a service, especially when wait and travel times could change from day to day depending on the passengers using the service, and the effects on medium-term demand. Can an equilibrium point be found between supply and demand? If too many people use the service, wait and travel times increase too much for it to be a feasible option for some passengers, and so they take other modes or decide not to travel. However, the then decreased demand leads to good level-of-service for the remaining passengers; other customers might then return, which leads to worsening level-of-service, and so on. Another case occurs when an initially demand-responsive service starts to become "regular" in its routes and timings, in which case it is more efficient to create a fixed-route service.

Using agent-based modelling, we explore how demand for DRT changes on a daily basis. Passengers make decisions about whether to use DRT to participate in an activity, using information and satisfaction levels from previous trips and publicly-available information about service levels. Results reported include the activity levels and locations of the population, as well as the performance of the transport network. Experimenting with a simplified environment shows that mismatched supply and demand lead to fluctuating performance of the DRT systems.

Keywords: Demand-responsive transportation, agent-based modelling, multi-day 


\section{INTRODUCTION}

Exploring multi-day demand for an transportation services is important to identify issues in catering for irregular and unpredictable demand. This is particularly important for on-demand transportation services, predominantly used for discretionary trips, such as shopping, leisure or visiting (Wang et al., 2012), which are not performed every day.

On-demand or demand-responsive transportation services differ from fixed-route public transport services by having fully-flexible or partially flexible routes and flexible timetables. While the cost and reliability of traditional public transport services are less likely to be affected by the number of passengers, services with dynamic routes could be affected if users are sporadic passengers or sparsely spatially distributed. This could lead to passengers choosing not to travel or to use another mode; it could also lead to inefficiencies for the operator.

The aim of this paper is to explore the use of DRT services over many days with iterative travel planning. In the past, DRT simulations have focused on single days or parts of days only; to the best of our knowledge, this is the first paper to consider individual activity patterns and interactions with DRT over many days. It is a preliminary study, focusing on the method for modelling many days, as well as demonstrating a simple scenario in order to explore the effects of multi-day modelling. Directions for further work are identified in the conclusion.

We consider two questions: firstly, what sort of patterns does DRT demand/usage form over many days, and secondly, does providing some form of average wait and travel times to the user make a difference to the demand and usage patterns.

Our first hypothesis is that demand for DRT is initially cyclical, in that as the service performance increases, more people are likely to take it, leading to decreased performance, meaning people will stop using it, and so on, before reaching some sort of equilibrium. Our second hypothesis is that the sharing of information between passengers, in this case indirectly via the operator, leads to more stable performance.

In Section 2, we review existing work on DRT, DRT simulation and modelling multi-day travel behaviour. Section 3 describes the model, while Section 4 describes the experimentation. Results are presented and discussed in Section 5. Conclusions and further work are discussed in Section 6.

\section{RELATED WORK}

A demand-responsive transportation service is a service somewhere between a bus and a taxi: it does not necessarily have a fixed route and timetable like a bus, however passengers travel together, and it can be booked like a taxi, by making a request to travel between two locations either immediately or at a future time.

Simulating demand-responsive transportation has been approached from multiple angles: optimisation, computer science and transportation (Ronald et al., 2015b). All viewpoints are required, from building schedules for vehicles, understanding passenger behaviour and simulating interactions and negotiations between passengers and operators.

Previous simulations have focused on one trip per person over a period of time, for example a few hours or a day. Neven et al. (2014) looked at forecasting DRT in the future, however did not consider DRT performance as a factor in their demand generation.

Moving away from modelling trips and short time periods leads to activity-based modelling, where the "what" and "why" of travel come into play. Modelling over many days is important as it provides an indication of the interactions between supply and demand. Ronald (2012) developed a multi-day model of joint activities generated via social networks. However, multi-day modelling is problematic in terms of data collection; most household travel surveys collect one day only in order to relieve respondent burden. Longer surveys do exist, for example Cirillo and Axhausen (2010) made use of a six-week diary.

An newer approach of approaching activity modelling is starting to consider the need for travel, that is, which needs would be satisfied by performing particular activities. The modelling of needs behind activity participation was demonstrated by Arentze and Timmermans (2009) and later refined by Nijland et al. (2009, 2010). This work identified six needs that drive participation in activities: social interaction, entertainment, new experience, relaxation, physical exercise, and fresh air and being outdoors. Activities completed could satsify one or more of these needs, which then could reduce the need for another activity.

In this work, we implement a simplified version of the needs-based model, as set out in Nijland et al. (2009): 
"an individual is faced with a decision to conduct an activity $i$ on a current day d given that the last time the activity was conducted was on day $\mathrm{s}<\mathrm{d}$ (this means that the time elapsed equals $\mathrm{d}-\mathrm{s}$ days)".

A point raised in Nijland et al. (2010) is that the mode of travel could satisfy a need, for example, cycling to an activity could reduce further need for physical exercise. This could also be the case for shared on-demand transportation: the social interaction of having to book via phone and share the vehicle with others has been noted as a benefit of the service ${ }^{1}$.

The needs approach provides a more realistic representation of travel demand, rather than randomly-assigned activities. It also fits nicely with the concept of DRT being used mostly for irregular or occasional needs.

\section{THE MODEL}

A model developed in MATSim (matsim.org, 2014) and based on the dvrp extension was used for this paper. The dvrp extension has previously been used for taxi routing (Maciejewski and Nagel, 2013). A previous implementation of DRT using this module was described in Ronald et al. (2015a), in which a single day of a fixed-timetable DRT service in regional Victoria, Australia was simulated and compared to an ad-hoc scheme.

Although MATSim is normally used iteratively, the dvrp module currently works with one run only. For this paper, scaffolding has been built around the single run implementation, which permits the creation of the input population and vehicle file for each day, the running of the model, and then the feedback to individuals.

\subsection{Entities and the environment}

There are two main entities: passenger agents who want to travel somewhere, and vehicles controlled by a driver (however could be autonomous in the future).

The passengers have a trip (or trips) that they want to make in order to do an activity, i.e., with an origin and destination, request time, expected frequency of the activity and a value of the activity. They also have a history of trips made, how much they "cost" (in terms of wait, travel time and price). They also have a strategy for estimating the cost of a trip, based on their history or some global information on wait and travel times.

Vehicles have a schedule of trips that they can execute. They operate over a street network containing links and nodes. Note that vehicles only stop at nodes; they cannot stop half-way along a link.

The environment contains some information on wait and travel times; this could be global, zonal, or for a particular street, and could be for one day or averaged over many days. Pricing can also be including in the model but was not included in the experimentation for this paper.

\subsection{Activity choice model}

The choice for passenger $i$ regarding whether to do an activity or not, given frequency $f_{i}$, days since last done $t_{i}$ (today - day last done), max activity value $a_{i}$ and threshold $u_{i}$, can be formulated as:

$$
V_{i}=\frac{a_{i}}{1+e^{\left(f_{i}-t_{i}\right)}}>u_{i}
$$

Note the choice is between doing activity and using DRT to get there, or not doing activity; no other modes are allowed. If the activity is completed, then the passenger earns the value of the activity has to spend time and money to get there; if activity is not completed, then the passenger does not receive the benefit of activity but also does not spend time nor money either. The needs-based approach (Equation 1) means value of activity increases over time; doing something you did the day before has lower value than something last done a week ago.

The probability of doing an activity on day $d, p_{i}^{a c t}(d)$, assuming doing the activity provides value $V_{i}$ and the expected cost of travel $\operatorname{cost}_{i}^{\text {exp }}=\mathrm{f}$ (wait time, travel time) when compared to doing nothing:

$$
\begin{aligned}
V_{i}^{a c t}(d) & =V_{i}-\operatorname{cost}_{i}^{e x p}(d)-u_{i} \\
p_{i}^{a c t}(d) & =\frac{e^{V_{i}^{a c t}(d)}}{1+e^{V_{i}^{a c t}(d)}}
\end{aligned}
$$

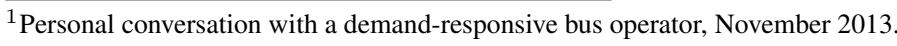


Note that $V_{i}$ is usually based on ongoing need, but $a_{i}$ is constant for each individual over the duration of the simulation. If $a_{i}$ is too low, then the activity is never done. In order to counter this, the initial setting of $a_{i}$ is related to the distance between the origin and destination, i.e., passengers with longer trips have a higher value.

Personal costs are then updated by $\operatorname{cost}_{i}(d)=(1-\alpha) \operatorname{cost}_{i}^{\text {trip }}(d)+\alpha \operatorname{cost}_{i}(d-1)$, however only if passenger $i$ travelled on day $d$. The generalised travel cost is updated after each trip, and is calculated by $\cos t_{i}^{\text {trip }}(d)=$ $2.0 \times t_{i}^{\text {wait }}(d)+t_{i}^{\text {travel }}(d)$. For all passengers, an average wait time $t_{\text {wait }}$ and excess travel factor $\beta_{\text {excess }}$ (i.e., distance actually travelled / direct distance) are provided at the end of each day; these could be calculated over one day or many days.

At this stage, the model only handles one-way trips, and ignores activity types. It could be that different activities lead to different tolerances in arrival times. Passengers cannot alter their departure time, which could lead to a better performance.

The choice to travel is effectively made the day before, rather than in real-time, hence the use of "expected cost". This is a simplification which could be explained by the need to make alternative arrangements if it looks like the service will not be practical. At this stage the model works with ad-hoc trips only, however can be extended to booking in advance (Ronald et al., 2015a).

\section{EXPERIMENTATION}

In terms of exploring the patterns, two perspectives are apparent: increasing demand by setting high activity values, and increasing supply by setting high numbers of vehicles. This produces four key scenarios:

1. high interest in travel and many vehicles, which should lead to reasonably comfortable travel;

2. low interest in travel and many vehicles, which should lead to low willingness to travel, but less sharing behaviour due to oversupply;

3. high interest in travel and few vehicles, which should lead to more sharing of trips;

4. low interest in travel and few vehicles, which should lead to quite constrained demand.

The environment is a $3 \mathrm{~km}$ by $3 \mathrm{~km}$ grid, with main streets every $1 \mathrm{~km}$ with $60 \mathrm{~km} / \mathrm{h}$ speed limits. Local streets run every $250 \mathrm{~m}$ with speed limits of $40 \mathrm{~km} / \mathrm{h}$.

For high interest in travel, the utility factor for $a_{i}$ was set at 5 ; for low, the limit was set at 2 . Scenarios with many vehicles contained 10 4-seater vehicles; few vehicle scenarios contained 2. In each run, there are 100 passengers, who make requests over a period of 59 minutes; the vehicles run for an extra 30 minutes to ensure everyone gets a ride. Activity thresholds were set to 0 and frequency to 1 for all passengers. $\alpha$ was set to 0.2 . The simulation was run for 300 days, and each scenario was repeated 10 times with different random seeds.

In terms of information, three scenarios were explored:

1. only personal data was used in making a decision to travel $($ local $) ; \operatorname{cost}_{i}^{\text {exp }}=\operatorname{cost}_{i}(d)$

2. only shared data (average wait times and excess travel factors over the past 7 days) was used (global); cost $_{i}^{\text {exp }}=2.0 \times t_{\text {wait }}+\beta^{\text {excess }} \times$ travel $_{i}^{\text {est }}$, where travel $_{i}^{\text {est }}$ is based on direct distance travelling at $50 \mathrm{~km} / \mathrm{h}$;

3. a combination of local and global, each weighted $50 \%$ (combined)

Outputs include:

- the number of individuals who used the service in the past 14 days (loyal passengers);

- the average number of passengers in the past 14 days;

- patronage for each day. 
Table 1. Summary statistics (loyal passengers / average daily passengers / range of daily passengers) for different scenarios.

\begin{tabular}{|l|c|c|c|c|}
\hline Vehicles & Few & Few & Many & Many \\
\hline Act. interest & Low & High & Low & High \\
\hline Local & $15.9 / 1.0 / 2.5$ & $27.2 / 10.9 / 7.9$ & $92.9 / 12.5 / 9.0$ & $98.9 / 59.8 / 13.8$ \\
\hline Combined & $60.0 / 6.8 / 8.6$ & $76.4 / 21.7 / 13.6$ & $99.2 / 26.2 / 15.0$ & $100.0 / 70.8 / 11.7$ \\
\hline Global & $91.7 / 13.1 / 15.0$ & $83.7 / 21.8 / 30.4$ & $100.0 / 42.0 / 15.0$ & $100.0 / 78.3 / 10.1$ \\
\hline
\end{tabular}

\section{RESUlts}

As a starting point, Table 1 shows the average number and range of passengers in the last 14 days of the simulation, and the number of unique passengers using the service in the last 14 days.

Using personal information only tends to lead to low patronage and loyalty - if passengers try the service once and do not like it, they have no further information for trying it again. The usage of service information leads to higher loyalty and higher patronage, however also leads to a larger variation in patronage, which could cause difficulties in planning supply. As expected, a combined strategy falls into an internediate place between local only and global only.

\subsection{Daily patronage fluctuation}

We are also interested in patterns of demand. This is more difficult to analyse as runs cannot be easily aggregated over time; the following results refer to run id 0 . In order to visually explore each run, the daily passenger totals were converted to a moving average for the previous 10 days, however subtracting the maximum and minimum value in those 10 days.

As seen from Table 1, using local information only provides low patronage when there is low interest in travelling and only a few vehicles. However, the scenarios with mismatched interest and supply (either high interest and few vehicles, or low interest and many vehicles) produce similar results in daily averages (10.9 vs 12.5). Figure 1 shows some fluctuation over time, which does not seem to reach any equilibrium point. For high interest and high demand, the fluctuation is reasonably stable.

However, with the inclusion of global information, we would expect more fluctuation. Also in Figure 1, daily passenger totals for combined and global scenarios are also presented. In comparison with the local information scenario, these fluctuate more.

\subsection{Spatial distribution}

Finally, a visual analysis of spatial distribution shows some clustering occuring in scenarios with no global information, in particular to the lower right of the environment (Figure 2). This could be occuring as the global information relates to the whole environment and entices passengers to try again.

\subsection{Discussion}

From the results, it can be seen that the provision of information makes a difference to the usage of a transportation service that has a route and timetable determined by demand. It can also be seen that demand for a flexible service is indeed fluctuating, depending on the behaviour of others, as well as the information provided as feedback from the system.

In reality, one would expect that passengers consider not only information provided by the operator, but also their own experiences, like the combined scenarios. Operators could use the global information as a form of marketing their services, however would have to be wary of raising expectations.

\section{CONCLUSION AND RECOMMENDATIONS}

Aiming for an exploratory simulation of the factors behind short-term DRT usage and an exploration of the interactions involved in demand, we showed that the provision of information, in this paper aggregate across the whole environment, does lead to better usage and performance of a flexible transportation system. Mismatched supply and demand, however, leads to fluctuations in performance; this observation points towards 


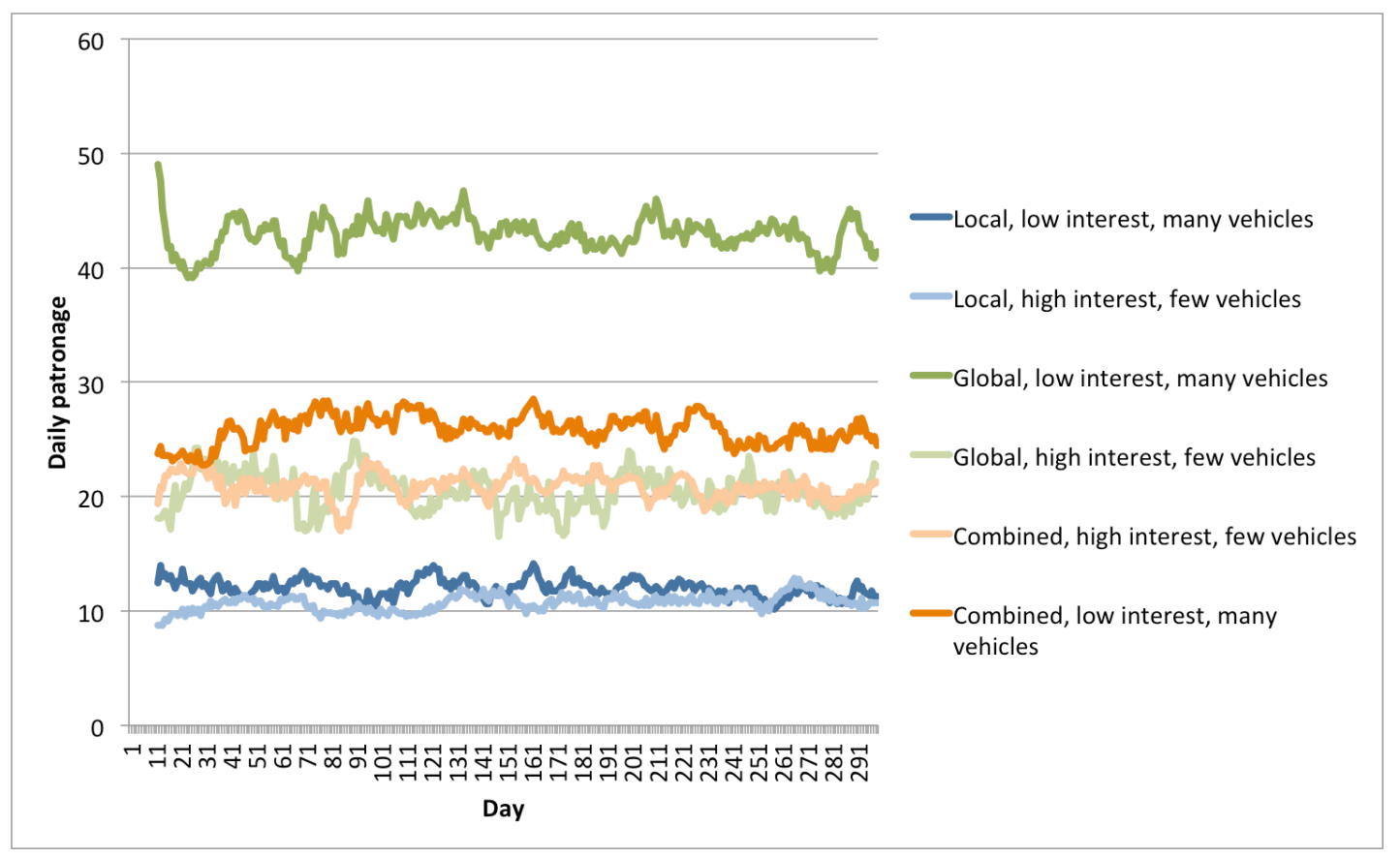

Figure 1. Daily patronage for selected local and global scenarios.

operators being able to make good decisions about patronage when planning supply, as well as passengers having useful information about the service.

This paper provides a proof-of-concept. Due to the small geographical area and the need for multi-day data, developing real-world inputs is not simple. Survey results from a multi-day activity survey could provide better indications of frequency in order to estimate parameters for different activities, as well as data from existing flexible transportation services. The effects of varied demand patterns can also be explored.

Another limitation is the single-mode choice, meaning individuals either do the activity or not; walking is not an option, nor is driving. Future work includes incorporating other modes. Adjusting the time of departure is also an extension; being able to recommend that others nearby are travelling around the same time could lead to more efficient performance for the operators and improved service for passengers. The addition of retry behaviour when only local knowledge is being utilised could also be useful, as well as the importance of sharing information via social networks. Ticket costs were also omitted from this model for simplicity, however can be easily incorporated. Finally, following comments by Nijland et al. (2010) regarding the mode itself as a satisfaction of needs, this could be explored further by taking into account the utility of shared travel as a satisfier of social needs.

\section{ACKNOWLedgements}

This work has been supported by a grant from the Australian Research Council (LP120200130).

\section{REFERENCES}

Arentze, T. A. and H. J. P. Timmermans (2009). A need-based model of multi-day, multi-person activity generation. Transportation Research Part B: Methodological 43(2), 251-265. 

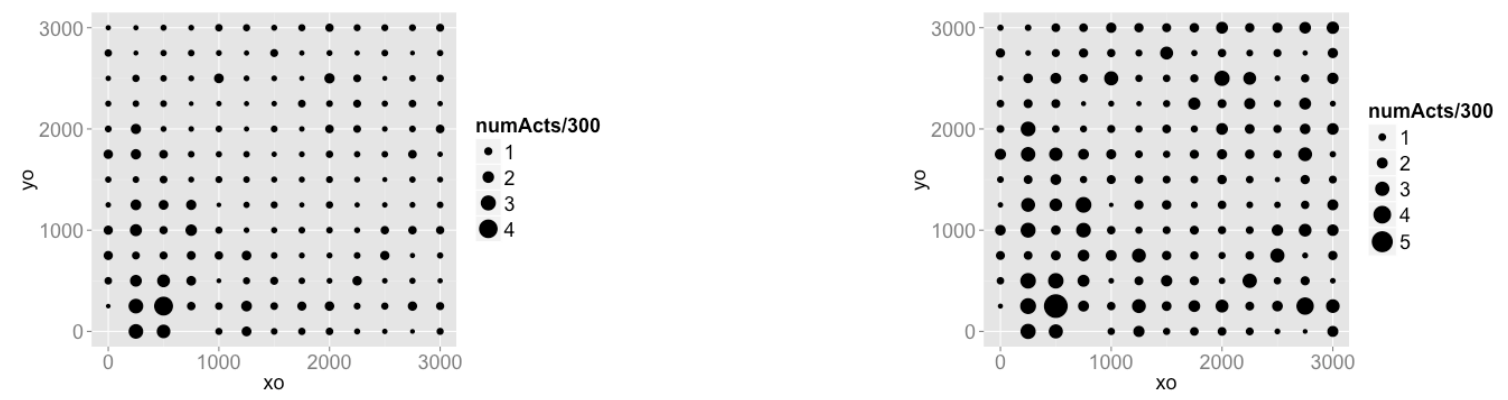

Figure 2. Origins of trips in local (left) and combined (right) scenarios with low interest and high vehicles.

Cirillo, C. and K. W. Axhausen (2010). Dynamic model of activity-type choice and scheduling. Transportation 37(1), 15-38.

Maciejewski, M. and K. Nagel (2013). Simulation and dynamic optimization of taxi services in MATSim. Technical Report 13-05, VSP, TU Berlin, Berlin, Germany.

matsim.org (2014). Agent-based Transport Simulations — MATSim.

Neven, A., K. Braekers, K. Declercq, T. Bellemans, D. Janssens, and G. Wets (2014). Methodology to optimize resource requirements of a demand responsive transport system for persons with disabilities: A case study of Flanders. In Transportation Research Board 93rd Annual Meeting, Washington, D.C., USA.

Nijland, L., T. Arentze, and H. Timmermans (2009). Estimating the Parameters of a Dynamic Need-Based Activity Generation Model. In Proceedings of the 12th International Conference on Travel Behaviour Research, Jaipur, India.

Nijland, L., T. Arentze, and H. Timmermans (2010). Eliciting the Needs That Underlie Activity-Travel Patterns and Their Covariance Structure: Results of Multimethod Analyses. Transportation Research Record 2157, 54-62.

Ronald, N. (2012). Modelling the effects of social networks on activity and travel behaviour. Phd thesis, Eindhoven University of Technology.

Ronald, N., R. G. Thompson, and S. Winter (2015a). A comparison of constrained and ad-hoc demandresponsive transportation systems. In Proceedings of the Transportation Research Board 94th Annual Meeting, Washington, DC, USA.

Ronald, N., R. G. Thompson, and S. Winter (2015b). Simulating demand-responsive transportation: a review of agent-based approaches. Transport Reviews 35(4), 404-421.

Wang, C., M. Quddus, M. Enoch, T. Ryley, and L. Davison (2012). The impact of socio-economic factors on the demand for demand responsive transport (DRT) in Greater Manchester: a multilevel modelling approach. In Transportation Research Board 91st Annual Meeting, Washington, DC, USA. 